\title{
Prototyping Retractable String-Based Interaction Techniques for Dual-Display Mobile Devices
}

\author{
Gábor Blaskó $^{\dagger}$, Chandra Narayanaswami ${ }^{\ddagger}$, Steven Feiner ${ }^{\dagger}$ \\ ${ }^{\dagger}$ Columbia University \\ Department of Computer Science \\ New York, NY, 10027 \\ \{gblasko,feiner\}@cs.columbia.edu \\ ${ }^{\ddagger}$ IBM TJ Watson Research Center \\ 19 Skyline Drive \\ Hawthorne, NY, 10532 \\ chandras@us.ibm.com
}

\begin{abstract}
Accessing information on mobile and wearable devices often requires the user's visual attention, and the precise operation of virtual or physical widgets. However, these interactions may sometimes be too time-consuming and socially inappropriate. To address this, we introduce a novel input/output device that is based on the manipulation of a retractable string in a polar coordinate frame. Depending on how the user pulls the string from its enclosure- to a particular length, at a particular angle - various system features may be directly accessed. Furthermore, we present our concept for a 1D pixel array, embedded in the string that may be used as a secondary 1D display. Since it is possible to unwind the display itself and trigger functionality with a single pull, information may be accessed and presented quickly, and perceived at a glance. We present scenarios for how the string input/output device may be used in conjunction with the mobile device's primary 2D display and describe our augmented reality proof-of-concept prototype.
\end{abstract}

\section{Author Keywords}

mobile interaction, mobile computing, wearable computing, retractable string-based input, pixel-array displays.

\section{ACM Classification Keywords}

H.5.2 User Interfaces, B.3.2. Input/Output Devices

\section{INTRODUCTION}

Small mobile and wearable electronic devices can be conveniently carried everywhere, allowing users to access information on the go anytime. Unfortunately, their applicability may be limited if user interaction consumes too much of the user's time and visual attention. In certain situations, only a few seconds of interaction may be socially permissible, giving the user barely enough time for a quickly executed input action (e.g., a button press) and a quick glance at the device's screen.

Permission to make digital or hard copies of all or part of this work for personal or classroom use is granted without fee provided that copies are not made or distributed for profit or commercial advantage and that copies bear this notice and the full citation on the first page. To copy otherwise, or republish, to post on servers or to redistribute to lists, requires prior specific permission and/or a fee.

CHI 2006, April 22-27, 2006, Montréal, Québec, Canada.

Copyright 2006 ACM 1-59593-178-3/06/0004...\$5.00.

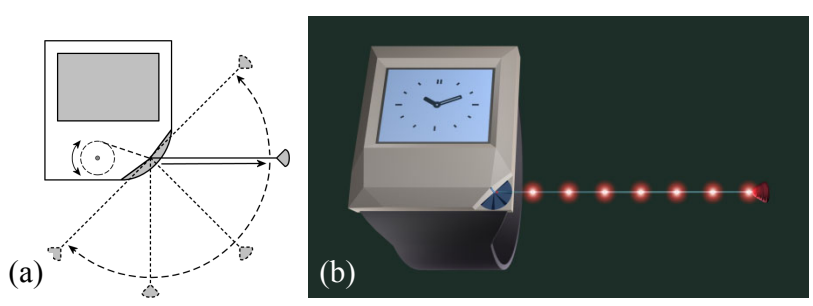

Figure 1. (a) Diagram of proposed retractable string device. (b) Concept rendering of 1D string display system, complementing the mobile device's primary $2 \mathrm{D}$ screen.

For each atomic system function to which the user may want immediate and direct access, a dedicated physical widget must be provided. But size constraints restrict the number and size of physical widgets with which small devices can be equipped. Furthermore, the smaller the physical widgets, the finer the motor skills required to operate them. In this paper, we introduce an input device that is based on the manipulation of a retractable string, wound onto a spool, that may be extremely small in size, but can still offer a large range of movement, thus moderating demands on motor skills (Figure 1a).

To present information on a portable device that may be understood in a quick glance, two approaches may be taken. If information is presented in detailed form, it may occupy a lot of screen space, in which case the device must be equipped with a sufficiently large screen to allow the user to skim the information with a single glance. Alternatively, if the device's display must be small, the information has to be presented more abstractly and compactly. On extremely small displays (e.g., those on watches), the physical density of the display may make the content difficult to understand at a glance. To address this problem, we introduce a compact display system formed by a linear array of colored lights integrated into the string device, which may be used to supplement the existing small graphical display of a portable or wearable device (Figure 1b).

In the remainder of this paper, we first introduce our novel input mechanism, based on the manipulation of a retractable string in a polar coordinate frame. Next, we extend this input device with 1D display capabilities and describe their joint use in a sample scenario. We then present the proofof-concept prototype we developed to demonstrate our ideas, using projector-based augmented reality (AR) [4]. 


\section{RELATED WORK}

Before a user can interact with many portable devices (e.g., a PDA or cell phone), the user must remove the device from its carrying place (e.g., a pocket or holster). Since this kind of device is typically held with one hand and its touchscreen or buttons operated with the other hand, both of the user's hands are often occupied during use. Then, once the interaction has ended, the device must be returned to its carrying place, to free up the user's hand.

In contrast, wearable computing devices are always readily available. Users may wear displays on their head or forearm, while input devices may be belt-mounted [3], or handheld [1]. We believe that the interaction and display system we introduce in this paper would be especially beneficial when used in conjunction with a wrist-worn computer [10].

Wrist-worn computers can be viewed with a quick glance; however, since they are strapped to the wrist, their interfaces must be operable with a single hand. With our proposed interaction device, if the spool on which the retractable string is wound is enclosed in a wrist-worn device, then the user may easily pull out the string with their other hand, and manipulate its end freely in space.

In our user interface, the position of the spool establishes the origin of the coordinate system in which the end of the string is manipulated. We believe that it is most natural to interact with this input mechanism in a polar coordinate frame. Comparable user interfaces are pie menus [5] and marking menus [9], found primarily in stylus-based user interfaces in which the point of initial contact establishes an origin relative to which the stylus is dragged. In pie menus and single-level marking menus, only the direction of the dragging gesture matters, and the length of the gesture/mark is irrelevant. In bullseye menus [6], menu items are distributed not only by angle but also by radial distance, and thus occupy connected angular segments. Inter-ring motion with a stylus or mouse in bullseye menus is performed without haptic feedback, requiring the user to precisely follow an angular trajectory [2], which may be time-consuming. In contrast, the string input device offers haptic feedback to guide the user, potentially assisting motion of the end of the string between neighboring angular segments within the same annular ring - by feeling the tension of the string, the user can keep the radial distance from the origin constant.

\section{RETRACTABLE STRING-BASED INPUT OVERVIEW}

The inspiration for our input interface is an item that is ubiquitous in corporate environments: a badge reel. These spring-loaded spools of string can be attached to belts or shirt pockets and hold an identity badge, often in a place that is easy for others to see. If the badge needs to be shown up close or swiped through a reader, the user can quickly move the badge to a nearby destination-without detaching it - and then return it to its original location with the help of the retracting mechanism. The entire sequence of events often takes a few seconds at most. Badge reels are small in size, and allow great freedom of movement for the badge in a way that is simple and intuitive. We decided to explore how this common mechanism might be exploited for input purposes on mobile and wearable devices.

Cable extension transducers (also known as yo-yo potentiometers, string potentiometers, and cable sensors) look much like badge reels, and are used to monitor the spatial displacement of objects by measuring the rotation of the transducer's spool. We have not been able to find any references to standalone cable extension transducers being used for human-computer interaction, other than for tracking. Vickers [11] tracked the end of a hand-held wand in 3D with three strings attached to ceiling-mounted shaft encoders. In Vectorix [8], the motion of a string is monitored in a spherical coordinate system with a custom built sensor to mechanically track the $3 \mathrm{D}$ position of the user's hand. In contrast, we are interested in experimenting with interaction techniques that explicitly use string length and pull angle in the user interface.

Rather than attempting to develop custom miniature sensors with which to experiment (e.g., an array of optical gates arranged in an arc to measure string angle), we decided on a different approach to building a proof-of-concept prototype to explore and illustrate the utility of such an input mechanism. Our functional prototype uses optical 3D tracking technology, external to the spool enclosure, to monitor the manipulation of the end of the retractable string. We attached a retroreflective marker to the end of a commodity badge reel, which we track in 3D with an Origin Instruments DynaSight optical radar system.

The input device is meant to be wrist-worn, and manipulated in free-space with a single hand. In our current prototype, the motion of the end of the string is monitored in a spherical wedge, spanning $30^{\circ}$, which is roughly parallel to the device's graphical display (Figure 2a). We subdivided this volume in which we track the motion of the end of the string into disjoint angular sections, which we call angular cells. As illustrated in Figure 2(b), we created a set of fifteen angular cells (five at each of three radial distances). The centers of these cells are angularly $45^{\circ}$ apart, spanning $\pm 10^{\circ}$, and radially $10 \mathrm{~cm}$ apart, spanning $\pm 3 \mathrm{~cm}$.

In bullseye [6] and pie menus [5], items - represented by angular segments - are adjacent, since the user may trigger menu item selection with an input mechanism (e.g., a button press) that is orthogonal to the spatial manipulation mechanism. The user may drag the cursor or stylus freely, moving from one segment to a neighboring segment, until one is activated by pressing a mouse button or lifting the

(a)

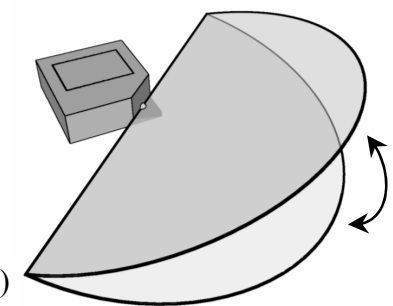

(b)

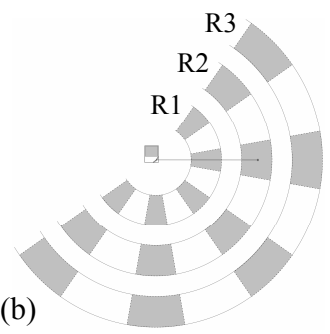

Figure 2. (a) Spherical wedge within which string is tracked. (b) Angular cells (shaded areas). 
stylus off the tablet surface. For our input system, we decided to explore the use of radial and angular motion alone to trigger events, forsaking the use of a button.

In our system, a function is triggered immediately when the end of the string is pulled into an angular cell, which has "buffer space" around it, as shown by the unshaded portions of Figure 2(b). However, we disable the activation of a cell when the string's end is moved into it from an outer radial ring during string retraction. The buffer space allows the user to manipulate the string in a larger volume than the activated angular cell itself, until a new cell is activated. In our prototype application, discussed later, the string input system serves to specify what is displayed on both the $2 \mathrm{D}$ display and the integrated string display. It does not control potentially destructive data manipulation, so there is no harm done if the user moves the end of the string into an angular cell by accident.

\section{STRING DISPLAY OVERVIEW}

Displays on wrist-worn devices are usually on the scale of a few square centimeters. Since the content must be legible, screens have to be designed not to be too visually dense, allowing the user to understand the content at a glance. Unfortunately, this means, for example, that in an appointment scheduling application, if only four or five short lines of text can be displayed at once, the user must scroll through multiple screens to get an overview of all events scheduled for a day. Alternatively, even if each appointment timeslot were to be presented more abstractly (e.g., by shading a rectangle to indicate whether it was occupied), the screen might be too dense because of the display's small physical size. In case of such an abstract representation of a timeline, the physical width of the screen would be too short to allow all the timeslot states to be shown along a single line. For example, on a $3 \mathrm{~cm}$ wide display, if the state of every half hour timeslot of an eight-hour workday were to be shown on a horizontal timeline, then each of the 16 timeslots would occupy less then $2 \mathrm{~mm}$ of the screen horizontally, resulting in a potentially incomprehensible pixel pattern.

A physical string display system could, in theory, be constructed from an array of individually addressable display elements (e.g., miniature color LEDs) positioned along the length of a retractable string. Since color LEDs as small as $0.5 \mathrm{~mm}$ in width are already available as surface-mount device components, we believe that in the near future it will be feasible to build this novel display system. To develop a proof-of-concept prototype now, we are using an approach similar to that of Holman et al. [7], who applied projectorbased AR [4] to explore interaction techniques for bendable paper displays. Our prototype projects colored patterns from above onto a tracked string, simulating an array of LEDs and projects a simulated 2D display onto the wrist, as shown in Figure 3. Even though the projected light is significantly dimmer than an array of LEDs, this provides an effective testbed in which to explore our UI concepts.

A retractable string display system has numerous benefits. The unwound string display may be an order of magnitude

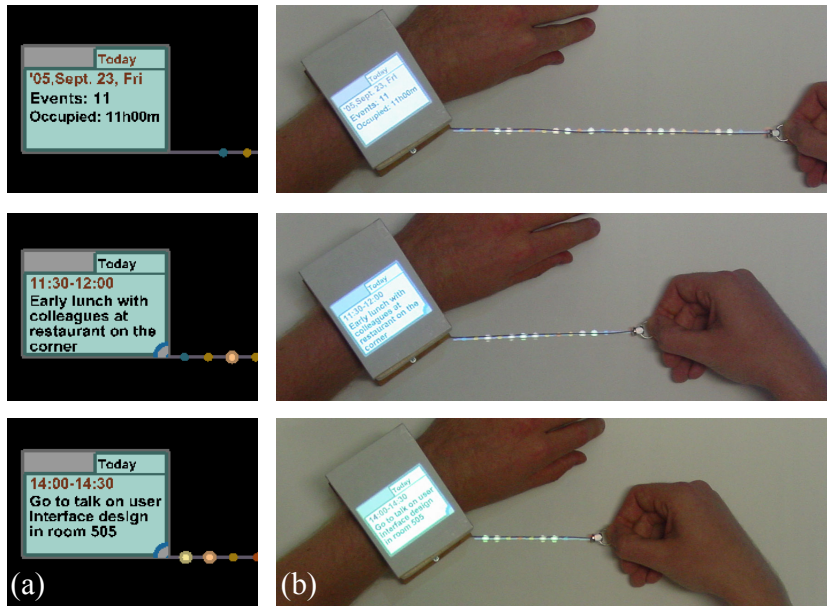

Figure 3. Appointment browser. (a) Screenshots of wristworn 2D display. (b) Projector-based AR prototype showing tracked string display.

longer than the largest dimension of the spool enclosure. This allows the individual display elements to be placed farther apart, thereby making the display less physically dense. Even if the display elements (e.g., LEDs) are single points, spacing them sufficiently far apart allows them to be visually differentiated far better than clusters of adjacent pixels on a tiny dense graphical display. By combining string input and display, new user interfaces can be created that complement the existing input and output capabilities of small devices. Next, we discuss a testbed application that illustrates some of the interaction techniques we are developing for the new device.

\section{PROTOTYPE SYSTEM OVERVIEW}

We wanted our testbed application to demonstrate how our string input/output system could be used to navigate amongst various information sources, and how a user could access information at different levels of detail using the string display with a primary 2D display. We chose three information sources that a user may wish to check multiple times throughout their busy day: their appointment schedule, incoming text messages, and stock portfolio data; these correspond to the three concentric rings (R1-R3) of Figure 2(b). We further subdivided each source into five subcategories: the schedule by days, text messages by sender information, and stocks by industry sector; these subcategories correspond to the five angular sections per ring.

As we had mentioned before, angular cells are activated only when the string is pulled outward, or when the end of the string is moved within a ring. Angular cells are spatially spread out and surrounded by buffers, allowing the user to activate a cell and change the display mode of the string display with a quick and relatively imprecise motion during a pull. When the string is pulled to a specific angular cell, an abstract representation of the data is displayed on the string display. Here, the user has the option to bring up the data in more detail on the 2D graphical display. This is done by manipulating the string precisely, as we discuss next. 
For example, if the user wishes to check how many messages they have received from family members, the user would pull the string out into the second ring (R2, $20 \mathrm{~cm}$ from the spool) horizontally east. At this point, the string display LEDs light up to show the number of newly arrived and unread messages. Each LED in the array represents a single message and may blink if the message is marked urgent. At a glance, the user is informed about the volume of new messages. At this point, the user has three choices.

- The contents of each message may be viewed on the portable device's 2D graphical display by retracting and repulling the string between the base state and the R2 ring. In this message browsing mode, when an LED of the retractable string is "swallowed" by the enclosure, the full text message, abstractly represented by that LED, is displayed on the 2D display. As long as the end of the string does not reach the next outer ring (i.e., R3) or the base state, free browsing of the messages is possible.

- From this cell, the user may move tangentially within the $\mathrm{R} 2$ ring to another cell to check how many messages have arrived from another category of senders (e.g., business clients), thus updating the number of string LEDs lit.

- The user may move the end of the string radially to the outermost ring R3, representing their schedule for the current day. Here, the LEDs become abstract representations of slots on a timeline. After the user has pulled the string to the R3 ring, changing the angle allows the user to browse their schedule for the other upcoming days.

In the appointment schedule mode (when the string is pulled out to $\sim 30 \mathrm{~cm}$ ), the string display shows a timeline of the user's day from 6 am to $6 \mathrm{pm}$, each LED representing a half-hour timeslot, as shown in Figure 3. Since the occupied timeslots are shown as illuminated LEDs, the user can get a quick overview of their day at a glance, and see if they have empty slots available. The user may see the details of a given appointment by retracting the string, metaphorically "popping" the time capsules onto the graphical display.

The combination of the retractable string and wrist-worn $2 \mathrm{D}$ display thus allows users to browse information at various levels of detail with a single fluid motion. Glanceable information is shown on the string display during the outward pull of the string and more detailed information is accessed on the traditional 2D display upon manipulating the string back and forth in a controlled manner, as long it does not reach the next outer ring.

\section{DISCUSSION AND FUTURE WORK}

Our projector-based AR approach to developing a proof-ofconcept prototype has allowed us to explore this new retractable string-based input/output device, without needing to build custom hardware. In the near future, we plan to extend our system by supporting full 3D wrist position- and orientation tracking, freeing users to try our system away from the tabletop, although still within a small projected volume. Since full 3D wrist tracking also enables us to determine vertical string angle, we will be able to test the util- ity of volumetric angular cells that are located in planes perpendicular to the plane of the device's primary graphical display.

We would ultimately like to build a hardware prototype with small cable extension transducers and an array of optical gate sensors to measure string angle. However, we first plan to conduct formal user performance studies with our current prototype. Our goal is to develop and validate a set of guidelines for effectively partitioning the polar coordinate frame. Using projector-based AR, we can test new configurations quickly and cheaply.

\section{ACKNOWLEDGEMENTS}

This research is funded in part by ONR Contract N0001404-1-0005, DAFAFRL Contract FA8650-05-2-6647, and gifts from IBM and Microsoft. The work reported in this paper is part of a Joint Study Agreement between IBM Research and Columbia University.

\section{REFERENCES}

1. Twiddler2 by HandyKey Corporation URL: http://www.handykey.com/

2. Accot, J. and Zhai, S., Beyond Fitts' law: Models for trajectory-based HCI tasks, Proc. CHI '97, 1997, pp. 295-302.

3. Bass, L., Kasabach, C., Martin, R., Siewiorek, D.P., Smailagic, A., and Stivoric, J., The design of a wearable computer, Proc. CHI '97, 1997, pp. 139-146.

4. Bimber, O. and Raskar, R., Spatial Augmented Reality: Merging real and virtual worlds, A. K. Peters Ltd., Wellesley, MA, 2004.

5. Callahan, J., Hopkins, D., Weiser, M., and Shneiderman, B., An empirical comparison of pie vs. linear menus, Proc. CHI'88, 1988, pp. 95-100.

6. Friedlander, N., Schlueter, K., and Mantei, M., Bullseye! When Fitts' law doesn't fit, Proc. CHI '98, 1998, pp. 257-264.

7. Holman, D., Vertegaal, R., Altosaar, M., Troje, N., and John, D., Paper windows: Interaction techniques for digital paper, Proc. CHI '05, 2005, pp. 591-599.

8. Kulik, A., Paneque, D., and Hochstrate, J., Vectorix: A Low-Tech Mechanical Tracking System, Proc. IEEE VR Workshop: Beyond Wand and Glove Based Interaction, 2004, pp. 25-27.

9. Kurtenbach, G., The design and evaluation of marking menus. PhD Thesis, Dept. of Computer Science, University of Toronto, 1993.

10. Narayanaswami, C. and Raghunath, M., Designing a new form factor for wearable computing, IEEE Pervasive Computing, 1(4), pp. 42-48, 2002.

11. Vickers, D., Sorcerer's apprentice: Head-mounted display and wand. Ph.D. Thesis, Department of Electrical Engineering, University of Utah, 1972. 